\title{
Monoamine Oxidase A is a Major Mediator of Mitochondrial Homeostasis and Glycolysis in Gastric Cancer Progression
}

This article was published in the following Dove Press journal: Cancer Management and Research

Ling Chen,' Li Guo, ${ }^{2}$ Ziwen Sun, ${ }^{3}$ Guochun Yang, ${ }^{4}$ Jing Guo, ${ }^{5}$ Kai Chen, $\mathbb{1}^{6}$ Ruixue Xiao,' ${ }^{7}$ Xigui Yang, ' Lijun Sheng'

'Department of Oncology, Affiliated Hospital of Shandong Academy of Medical Sciences, Shandong First Medical University, Jinan, Shandong, People's Republic of China; ${ }^{2}$ Department of Clinical Laboratory, Affiliated Hospital of Shandong Academy of Medical Sciences, Shandong First Medical University, Jinan, Shandong, People's Republic of China; ${ }^{3}$ Department of Scientific Research and Education, Affiliated Hospital of Shandong Academy of Medical Sciences, Shandong First Medical University, Jinan, Shandong, People's Republic of China; ${ }^{4}$ Department of Emergency Medicine, Affiliated Hospital of Shandong Academy of Medical Sciences, Shandong First Medical University, Jinan, Shandong, People's Republic of China; ${ }^{5}$ Department of Medical Oncology, Xiamen Key Laboratory of Antitumor Drug Transformation Research, The First Affiliated Hospital of Xiamen University, Xiamen, Fujian, People's Republic of China; ${ }^{6}$ The Department of Cardiovascular and Thoracic Surgery, The Second Affiliated Hospital and Yuying Children's Hospital of Wenzhou Medical University, Wenzhou, Zhejiang, People's Republic of China; ${ }^{7}$ Department of Pathology, Affiliated Hospital of Shandong Academy of Medical Sciences, Shandong First Medical University, Jinan, Shandong, People's Republic of China

Correspondence: Lijun Sheng Email shenglijun328@I26.com
Objective: Monoamine oxidase A (MAO-A) is a mitochondrial protein involved in tumourigenesis in different types of cancer. However, the biological function of MAO-A in gastric cancer development remains unknown.

Methods: We examined MAO-A expression in gastric cancer tissues and in gastric cancer cell lines by immunohistochemistry and Western blot analyses. CCK8, FACS and bromodeoxyuridine incorporation assays were performed to assess the effects of MAO-A on gastric cancer cell proliferation. The role of MAO-A in mitochondrial function was determined through MitoSOX Red staining, ATP generation and glycolysis assays.

Results: In the present study, we observed that MAO-A was significantly upregulated in gastric cancer tissues and in AGS and MGC803 cells. The observed MAO-A inhibition indicated decreased cell cycle progression and proliferation. Silencing MAO-A expression was associated with suppressed migration and invasion of gastric cancer cells in vitro. Moreover, alleviated mitochondrial damage in these cells was demonstrated by decreased levels of mitochondrial reactive oxygen species and increased ATP generation. MAO-A knockdown also regulated the expression of the glycolysis rate-limiting enzymes hexokinase 2 and pyruvate dehydrogenase. Finally, we observed that the glycolysis-mediated effect was weakened in AGS and MGC803 cells when MAO-A was blocked.

Conclusion: The findings of the present study indicate that MAO-A is responsible for mitochondrial dysfunction and aerobic glycolysis, which in turn leads to the proliferation and metastasis of human gastric tumour cells.

Keywords: monoamine oxidase A, mitochondrial dysfunction, gastric cancer, glycolysis

\section{Introduction}

Although numerous improvements have been made in gastric cancer (GC) diagnosis and treatment, GC still is the third most deadly cancer worldwide, especially in East Asia. ${ }^{1-4}$ Thus, the further identification and validation of GC-associated genes, new pathways and therapeutic targets, drugs and strategies for combination therapy are urgently needed.

There is a growing body of evidence suggesting that GC cells preferentially rely on aerobic glycolysis to generate energy rather than mitochondrial respiration. ${ }^{5}$ Accelerated glucose uptake in GC cells during aerobic glycolysis has been shown to be regulated by hexokinase II (HK II), pyruvate kinase (PKM), pyruvate dehydrogenase (PDH), phosphofructokinase-1 (PFK-1) and lactate dehydrogenase (LDHA). ${ }^{6,7}$ Metabolic reprogramming has been identified as a hallmark of GC and 
is required for $\mathrm{GC}$ cell survival and metastases. ${ }^{8,9}$ However, the underlying molecular mechanism associated with aerobic glycolysis and metabolic reprogramming during GC differentiation and metastasis remains unclear.

Mitochondria are essential regulators that serve as energy factories in cells through oxidative phosphorylation. $^{10,11}$ Mitochondrial damage with respect to electron transport chain carriers, mitochondrial DNA (mtDNA), calcium levels and lipid homeostasis as well as metabolic reprogramming have been reported in various cancers. ${ }^{12,13}$ However, although dysfunctional mitochondria are associated with the development of GC, the key mitochondrial regulatory mechanisms remain to be elucidated. ${ }^{14}$ Monoamine oxidase-A (MAO-A) exists in the mitochondrial outer membrane and preferentially degrades biogenic amines including serotonin, norepinephrine and epinephrine. ${ }^{15}$ MAO-A activity has been shown to be associated with anxiety, panic disorder, impulsivity, autism and antisocial behaviour in humans. ${ }^{16-18}$ The results of recent studies have shown abnormal expression of MAO-A in prostate cancer and hepatocellular carcinoma, suggesting a novel role of MAO-A in mediating tumour progression. ${ }^{19,20}$ However, the biological activities of MAO-A in GC and the specific role of MAO-A with respect to aerobic glycolysis or mitochondrial respiration remain largely unknown. Therefore, in the present study, we aimed to determine the precise effects of MAO-A in the mitochondrial metabolic reprogramming of $\mathrm{GC}$ cells and to elucidate the role of MAO-A in regulating various GC cellular processes, including proliferation, migration and invasion.

\section{Materials and Methods}

\section{Clinical Samples}

Primary GC samples (106 cases) and corresponding normal tumour adjacent specimens (92 cases) were obtained from the pathology department of Affiliated Hospital of Shandong Academy of Medical Sciences between August 2008 and July 2013. The patients included 70 males and 36 females, aged 26 to 83 years, with an average age of 62 years. All cases were confirmed by pathological diagnosis with complete clinical and followup data. The clinicopathological indicators included the age, gender, general classification, histologic subtype, Lauren classification, tumour size, lymph node metastasis, vascular invasion, TNM staging, and survival status of the patients. Survival time was calculated from the date of surgery to the date of the last follow-up or the date of death due to recurrence/metastasis. None of the patients had received any systematic preoperative radiotherapy, chemotherapy or other antitumour therapy. The present study was approved by the Ethics Committee of the Affiliated Hospital of Shandong Academy of Medical Sciences and was conducted in accordance with the 1964 Helsinki declaration. Signed informed consent was obtained from each patient.

\section{Immunohistochemistry}

All specimens used for immunochemical staining were fixed in $4 \%$ formalin, embedded in paraffin wax and cut into 5 - $\mu \mathrm{m}$ thick-sections. After being deparaffinized and rehydrated in graduated alcohol, the sections were incubated with primary antibodies against MAO-A (diluted 1:100, ab126751, Abcam, UK), PDH (diluted 1:100, 3205, Cell Signaling Technology, USA) and HK II (diluted 1:100, 2867, Cell Signaling Technology, USA), overnight at $4{ }^{\circ} \mathrm{C}$ and then were treated with a secondary antibody at room temperature. The sections were visualized by treatment with a 3.3-diaminobenzidine solution for $3 \mathrm{~min}$ followed by counterstaining with haematoxylin.

The immunostaining analysis was performed independently in a blinded manner by two experienced pathologists, who selected three fields at high magnification $(400 \times)$ and counted 100 to 200 cells in each field. The expression of MAO-A, HK II and PDH was evaluated according to positively stained cells and staining intensity. Positive staining was scored as $0(0 \%), 1$ (1 25\%), 2 (26 50\%), $3(51 \sim 5 \%)$, and $4(76 \sim 100 \%)$ and the staining intensity was scored as 0 (negative), 1 (weak), 2 (medium) or 3 (strong). The final scores were calculated as a product of the positive staining score multiplied by the staining intensity score. We defined tissue samples with a score $\geq 4$ as exhibiting high expression, and those with a score $<4$ as exhibiting low expression.

\section{Cell Culture}

The human gastric cancer cell lines MGC803, NCI-N87, and AGS, and the immortalized normal gastric epithelial cell line GES-1 (purchased from the Procell Life Science Technology Co., Ltd) were used in the present study. Cells were cultured in RPMI-1640 medium containing 10\% foetal bovine serum (FBS) supplemented with $100 \mathrm{U} / \mathrm{mL}$ of penicillin and $100 \mu \mathrm{g} / \mathrm{mL}$ of streptomycin according to the manufacturer's instructions. Cells were maintained at $37{ }^{\circ} \mathrm{C}$ under a humidified atmosphere with $5 \% \mathrm{CO}_{2}$. 


\section{Cell Proliferation Assay}

Cell proliferation was assessed using a Cell Counting Kit8 (Beyotime, C0038, Shanghai, China). Cells were cultured in a 96-well plate at a density of $1 \times 10^{4} /$ well and then treated with different agents. After incubating for $24 \mathrm{~h}$ at $37{ }^{\circ} \mathrm{C}$, the WST-8 (C20H13N6NaO11S2) solution $(5 \mu \mathrm{L})$ was added into each well. Then, after incubating for $2 \mathrm{~h}$ at $37{ }^{\circ} \mathrm{C}$, the optical density (OD) was measured using a spectrophotometer at a wavelength of $450 \mathrm{~nm}$.

\section{5-Bromodeoxyuridine (BrdU) Incorporation}

Cells were plated in 96-well plates at a density of 5000/ well and then treated with different agents in RIPM supplemented with $10 \% \mathrm{FBS}$ and $10 \mathrm{ng} / \mathrm{mL}$ of BrdU labelling solution for $24 \mathrm{~h}$. BrdU incorporation was measured according to the Millipore BrdU proliferation assay kit instructions (Merck, 2750, Darmstadt, Germany). Briefly, cells were incubated for $1 \mathrm{~h}$ with an anti-BrdU monoclonal antibody. Then, the cells were washed and incubated with peroxidase goat anti-mouse IgG at room temperature. Subsequently, $100 \mu \mathrm{L}$ of substrate was added to each well and incubated for $30 \mathrm{~min}$ in darkness. Finally, after adding $100 \mu \mathrm{L}$ stop solution, the optical density (OD) was detected with a spectrophotometer at the wavelength of $450 \mathrm{~nm}$.

\section{Measurement of Mitochondrial Reactive Oxygen Species (ROS)}

Cells were cultured in different agents for $24-48 \mathrm{~h}$ before being loaded with $20 \mu \mathrm{M}$ MitoSOX Red (Thermo Fisher, M7513) and incubated for $30 \mathrm{~min}$ at $37^{\circ} \mathrm{C}$. After washing the cells three times with PBS, the accumulation of mitochondrial ROS (red) was visualized by confocal microscopy.

\section{siRNA Design and Transfection}

To silence the expression of MAO-A protein, cells were transfected with the small interfering RNA (siRNA), which was designed and synthesized by GenePharma (Suzhou, China). Nontargeted control siRNA (siNC) was used as the negative control. The sense sequence of siRNA against MAO-A and the non-targeted control sequence are as follows: MAO-A:5'-GCUGAACCAUGAACAUUAUT T-3', and negative control (NC): 5'-UUCUCCGAACG UGUCACGUTT- $3^{\prime}$. The cells were cultured to $70-80 \%$ confluence, after which $5 \mu \mathrm{L}$ of siRNA and $5 \mu \mathrm{L}$ of
X-tremeGENE siRNA transfection reagent were diluted in the RPMI-1640 medium for $5 \mathrm{~min}$. Then, after mixing them together, the mixture (siRNA/transfection reagent) was incubated at room temperature for $18 \mathrm{~min}$ before being added to cells. After transfection, the cells were quiescent for $24 \mathrm{~h}$ and then used as required.

\section{Cell Cycle and DNA Analysis}

A Cell Cycle and Apoptosis Analysis Kit were used to examine whether the cell cycle was influenced in the different experimental groups. Cells were centrifuged and then resuspended in $1 \mathrm{~mL}$ of cold PBS. Subsequently, following two washes with PBS, the cells were resuspended and fixed with $70 \%$ ice-cold ethanol at $4{ }^{\circ} \mathrm{C}$ overnight. Then the ethanol was removed and the cells were incubated in $200 \mu \mathrm{L}$ of PBS. The cells were then stained with propidium iodide (Beyotime, C1052, Shanghai, China) according to the protocol provided with the kit, and cell cycle analysis was performed by fluorescence flow cytometry on a BD FACSCalibur flow cytometer.

\section{Detection of Cellular ATP Levels}

The level of ATP in cells was analysed using an ATP Bioluminescence Assay kit (Beyotime, S0026, Shanghai, China). Briefly, cells were lysed with a lysis buffer, and then centrifuged at $12,000 \mathrm{rpm}$ for $2 \mathrm{~min}$ at $4{ }^{\circ} \mathrm{C}$. The level of ATP was determined by mixing $50 \mu \mathrm{L}$ of the supernatant with $50 \mu \mathrm{L}$ of luciferase reagent where the emitted light was linearly related to the ATP concentration and measured using a microplate luminometer according to the standard protocol described in the ATP assay kit.

\section{Mitochondrial Glycolysis Assays}

Mitochondrial glycolysis was analysed using a Seahorse XF24 Extracellular Flux Analyzer (Seahorse Biosciences, Billerica, MA, USA) by real-time measurements of the extracellular acidification rate (ECAR). Cells were plated at 6000 cells/well in XF24 culture microplates. Then, the cells were glucose-starved in XF assay medium in a CO2free XF prep station at $37{ }^{\circ} \mathrm{C}$ for $60 \mathrm{~min}$, where glucose $(2 \mathrm{mg} / \mathrm{mL})$, oligomycin $(1 \mu \mathrm{M})$ and 2-deoxy-D-glucose (2-DG, $100 \mathrm{mM})$ were automatically injected into the instrument.

\section{Wound Healing Assay}

Cells were cultured in six-well plates and upon reaching $80 \%$ confluency, the monolayer was wounded with a yellow pipette tip (1-mm width). Then, the wounded 
cells were examined under an inverted microscope at 0 $\mathrm{h}$ and received various treatments. After $24 \mathrm{~h}$, images were taken in the same areas as those recorded at $0 \mathrm{~h}$.

\section{Migration and Invasion Assays}

Cell migration was assessed using a modified Boyden chamber (Corning) where 10\% FBS was added to the lower layer of the transwell as a chemoattractant and cells resuspended in RPMI-1640 medium without FBS were added to the upper chamber. Subsequently, the cells that adhered to the 8 - $\mu \mathrm{m}$-pore polycarbonate filter were fixed with $4 \%$ paraformaldehyde, and stained with $0.4 \%$ crystal violet. Then, cells in the upper portion of the filter were removed, and the number of stained migrated cells was counted under an inverted microscope.

The invasion assay was also performed using a modified Boyden chamber, where the filters were filled with $50 \mu \mathrm{g} / \mathrm{mL}$ of Matrigel solution (BD Biosciences), and the lower chamber was filled with $10 \%$ FBS. After incubating for $24 \mathrm{~h}$, the cells in the Boyden filters were removed from the lower chamber, and then stained with crystal violet solution. Images were captured using a microscope.

\section{Western Blot Analysis}

Samples containing $20 \mu \mathrm{g}$ of total proteins were heated with SDS-PAGE sample buffer for $5 \mathrm{~min}$ at $100{ }^{\circ} \mathrm{C}$ and then separated in $10 \%$ or $12 \%$ SDS-PAGE gels. The proteins were then transferred to nitrocellulose membrane and incubated with Tris-buffered saline $(20 \mathrm{mM}$ Tris, $150 \mathrm{mM}$ $\mathrm{NaCl}$ and $0.1 \%$ Tween $20, \mathrm{pH}=7.6$ ) containing 5\% nonfat dry milk for $1 \mathrm{~h}$. Subsequently, the membranes were incubated initially with antibodies directed against MAOA (1:1000), cyclin A and D (1:400, Cell Signaling Technology, USA), PDH (1:1000) and HK II (1:500) overnight at $4^{\circ} \mathrm{C}$, followed by sequential incubations with a HRP-conjugated secondary antibody (1:5000-10000) and enhanced chemiluminescence reagents, with $\beta$-actin serving as an internal control.

\section{Data Analysis}

The data are presented as the means \pm SEM. Statistical analysis was performed with Student's $t$-test or one-way ANOVA followed by Tukey's test where appropriate. Differences where $\mathrm{P}<0.05$ were considered significant. The $\chi^{2}$ test was used to analyse the associations of protein expression with clinicopathologic features. Survival analysis was performed using the Kaplan-Meier method, and the difference in survival between the groups at each observed time point was compared by the Log rank test.

\section{Results}

\section{MAO-A Expression is Increased in GC} Tissues and in Cultured GC Cell Lines

The results of a previous study indicated that altered glucose metabolism through enhanced aerobic glycolysis (known as the Warburg effect) distinguishes cancer from normal tissues. ${ }^{21}$ To explore the role of MAO-A in GC with respect to glycolysis, the expression of MAO-A and two key glycolytic enzymes (HK II and PDH) was first evaluated in paired GC samples by immunohistochemical analysis (Figure 1A). The correlations between MAO-A, HK II and PDH expression with clinical and pathological characteristics of GC patients are summarized in Table 1. The expression levels of MAO-A and HK II in GC tissues were $56.6 \%(60 / 106)$ and $47.2 \%(50 / 106)$, respectively, while the rates of high MAO-A and HK II expression in the corresponding paracancerous gastric mucosa were $26.1 \%$ (24/92) and 22.8\% (21/92). The rate of high PDH expression in GC tissues was only $32.1 \%$ (34/106), while that observed in the high expression rate of $\mathrm{PDH}$ in the corresponding paracancerous tissues was 53.3\% (49/92), showing a significant difference $(\mathrm{P}=0.003)$. Spearman correlation results showed that the expression of MAO-A was positively correlated with HK II levels $(r=0.616, \mathrm{P}<0.001)$ but negatively correlated with $\mathrm{PDH}$ expression $(\mathrm{r}=-0.301$, $\mathrm{P}=0.002$ ) (Tables 2-3). The survival analysis of $\mathrm{GC}$ patients further revealed that high $\mathrm{PDH}$ expression was associated with poor survival (Figure 1B). To further assess the expression of MAO-A in vitro, we performed Western blot analysis using various gastric cancer cell lines including AGS, NCL-N87 and MGC803. Increased MAO-A protein levels were observed in gastric cancer cells compared to those observed in gastric epithelial GES-1 cells, and especially in AGS and MGC803 cells, prompting us to perform subsequent experiments using AGS and MGC803 cells (Figure 1C).

\section{Cell Proliferation and DNA Synthesis are Associated with the MAO-A Pathway}

To ascertain the role of MAO-A in gastric cancer cell function, we first silenced MAO-A expression in the GC cell lines AGS and MGC803. The efficacy of the silencing effect was confirmed as shown in Figure 2A and B. Then, $\mathrm{GC}$ cell proliferation was assessed by $\mathrm{CCK} 8$ and $\mathrm{BrdU}$ 


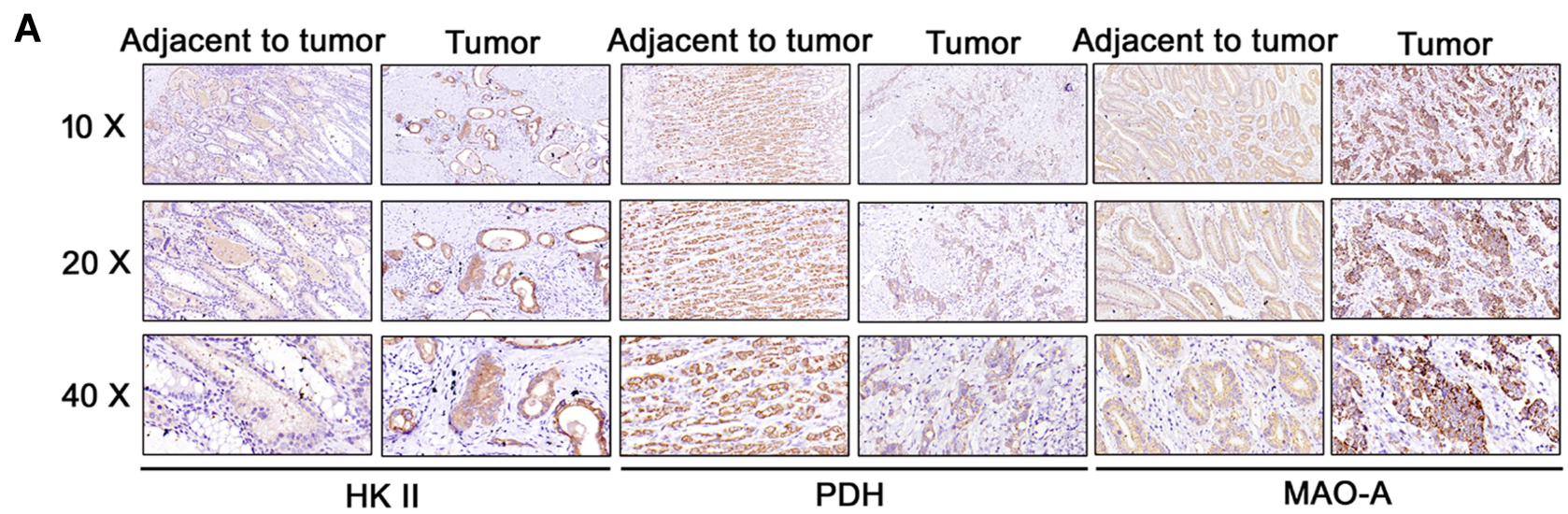

B

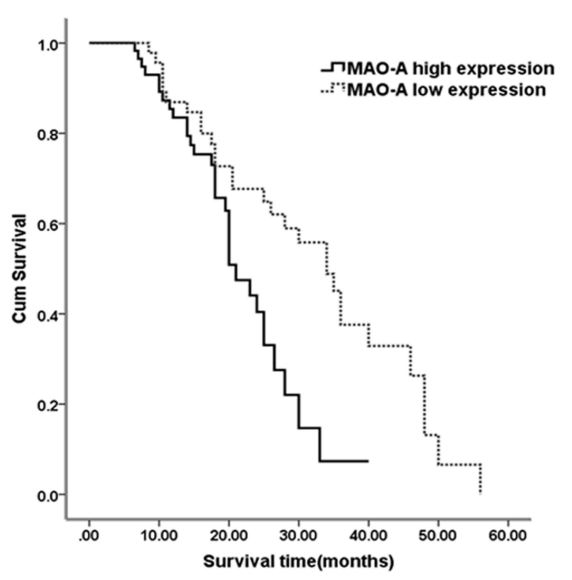

C

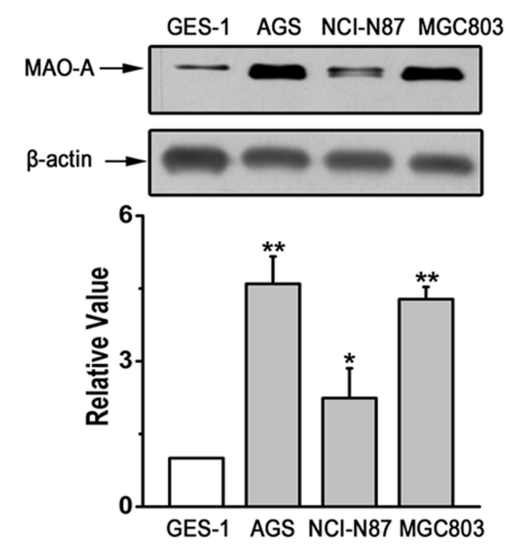

Figure I Expression of MAO-A in vivo and in vitro. (A). Representative immunohistochemical staining of HK II, PDH and MAO-A in GC tumour and paracancerous tissues. (B). Survival curve of MAO-A expression in gastric cancer patients. (C). Expression of MAO-A in cultured GES-I and GC cell lines. $n=4$. *P $<0.05$, **P $<0.01$ compared with GES-I. All of the values are presented as the means \pm SEM.

Abbreviations: AGS, MGC803, and NCI-N87, human gastric cancer cell lines; GC, gastric cancer; GES-I, gastric epithelial cell line; HK II, hexokinase II; MAO-A, monoamine oxidase-A; PDH, pyruvate dehydrogenase.

incorporation assays. The results showed that silencing MAO-A (siMAO-A) expression significantly inhibited AGS and MGC803 cell viability (Figure 2C-D). Moreover, decreased DNA synthesis was observed after MAO-A knockdown, indicating that MAO-A is a positive regulator of $\mathrm{GC}$ cell proliferation (Figure $2 \mathrm{E}-\mathrm{F}$ ).

\section{MAO-A Regulates Expression of Cell Cycle-Related Proteins and Mediates Cell-Cycle Progression}

To investigate whether MAO-A affects the cell cycle regulation of GC cells, we performed cell cycle analysis by flow cytometry. As shown in Figure 3A, siMAO-A remarkably decreased the percentages of AGS cells in the $\mathrm{S}$ and $\mathrm{G}_{2} / \mathrm{M}$ phases compared with that observed in the NC group. Similar results were observed for MGC803 cells, with more cells to remaining in the $G_{0} / G_{1}$ phase after
MAO-A siRNA treatment (Figure 3B). Since cyclin A and $D$ play important roles in the $S$ and $G_{2} / M$ phases of the cell cycle, we analysed the expression of these proteins in GC cells. A significant reduction in the posttranslational levels of these cell cycle regulated proteins was observed after knocking down MAO-A (Figure 3C-D). These results suggest that $\mathrm{MAO}-\mathrm{A}$ is involved in the cell cycle control of GC cells.

\section{Migration and Invasion of GC Cells are Inhibited by Knocking Down the MAO-A Gene}

In addition, we determined the role of MAO-A in GC cell migration and invasion. The scratch wound assay results indicated that siMAO-A produced a significant inhibition of the migratory capability after $24 \mathrm{~h}$ in AGS (Figure 4A) and MGC803 cells (Figure 4B). Correspondingly, in the 


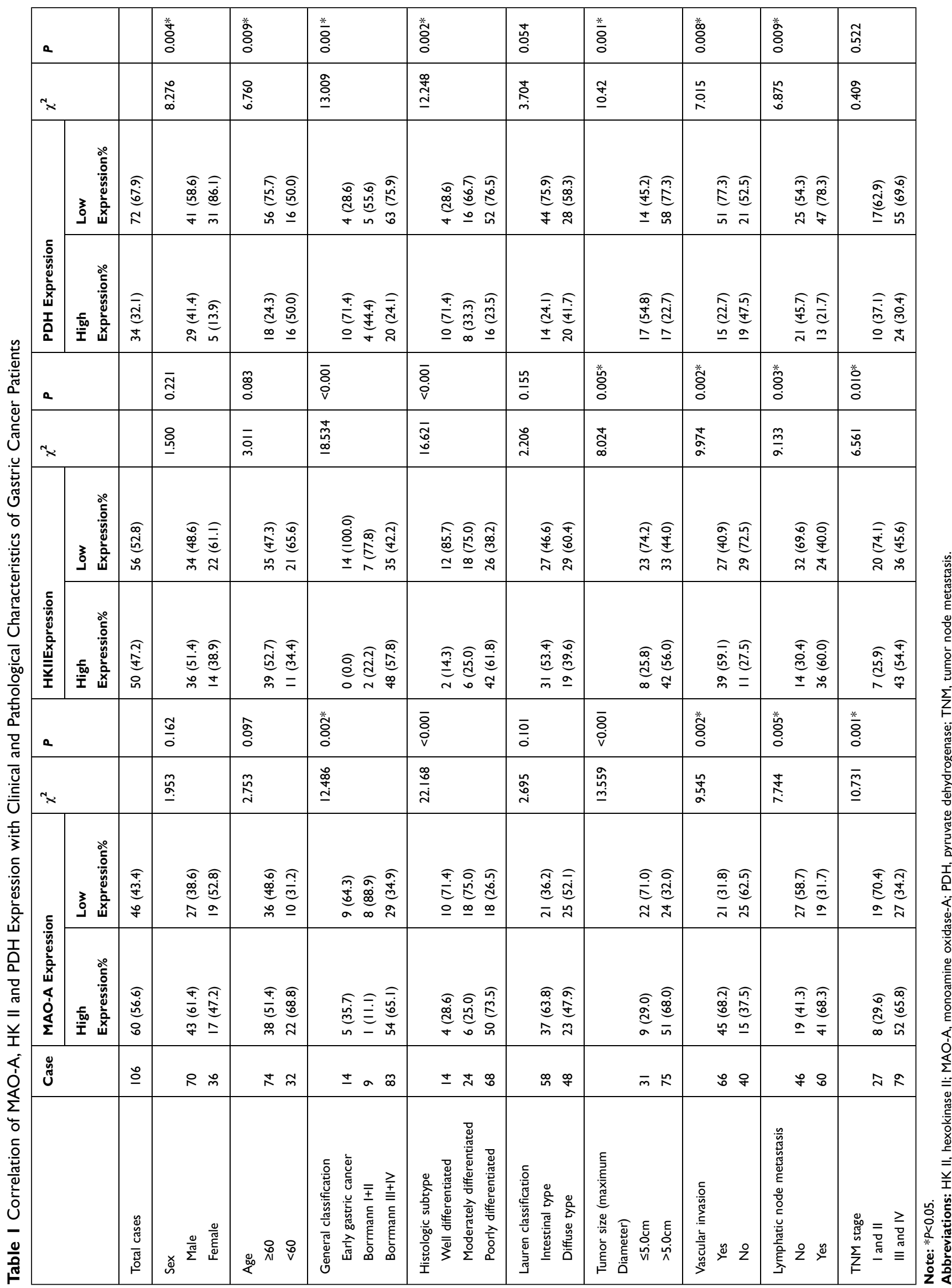


Table 2 Correlation Analysis of MAO-A and HK II Expression

\begin{tabular}{|l|l|l|l|l|}
\hline MAO-A & \multicolumn{2}{|l|}{ HK II } & $\boldsymbol{r}$ & $\boldsymbol{P}$ \\
\cline { 2 - 5 } & $\begin{array}{l}\text { High } \\
\text { Expression }\end{array}$ & $\begin{array}{l}\text { Low } \\
\text { Expression }\end{array}$ & $\mathbf{0 . 6 1 6}$ & $<\mathbf{0 . 0 0 I}$ \\
\hline High expression & 36 & 24 & & \\
Low expression & 14 & 32 & & \\
Total number & 50 & 56 & & \\
\hline
\end{tabular}

Abbreviations: HK II, hexokinase II; MAO-A, monoamine oxidase-A.

Table 3 Correlation Analysis of MAO-A and PDH Expression

\begin{tabular}{|l|l|l|l|l|}
\hline MAO-A & \multicolumn{2}{|l|}{ PDH } & $\boldsymbol{r}$ & $\boldsymbol{P}$ \\
\cline { 2 - 5 } & $\begin{array}{l}\text { High } \\
\text { Expression }\end{array}$ & $\begin{array}{l}\text { Low } \\
\text { Expression }\end{array}$ & $-\mathbf{0 . 3 0 1}$ & $\mathbf{0 . 0 0 2}$ \\
\hline High expression & 12 & 22 & \\
Low expression & 48 & 24 & & \\
Total number & 60 & 46 & & \\
\hline
\end{tabular}

Abbreviations: MAO-A, monoamine oxidase-A; PDH, pyruvate dehydrogenase.

transwell experiments, MAO-A deficiency dramatically suppressed the migration of GC cells (Figure 4C). Moreover, silencing of MAO-A gene expression in AGS and MGC803 cells also had an obvious inhibitory effect on cell invasion (Figure 4D). Therefore, these results indicate the crucial role of MAO-A in promoting GC metastasis.

\section{MAO-A Regulates Mitochondrial Function in AGS and MGC803 Cells}

Based on the observations described above, it is possible that MAO-A plays an important role in GC malignant process. However, the underlying mechanism associated with this process has not been demonstrated, and it is possible that this activity results from mitochondrial dysfunction. To determine whether mitochondrial homeostasis is mediated by MAO-A in GC metastasis, mitochondrial reactive oxygen species (ROS) levels were first analysed using MitoSOX Red Mitochondrial Superoxide Indicator. As shown in Figure 5A, siMAO-A decreased mitochondrial ROS generation in both AGS and MGC803 cells. Furthermore, there was a significant increase in ATP generation by $\mathrm{GC}$ cells after MAO-A inhibition (Figure 5B-C), suggesting that MAO-A plays a crucial role in regulating mitochondrial function.

\section{MAO-A Regulates Glycolysis in GC}

The mitochondrial metabolic switch from oxidative phosphorylation to glycolysis is crucial for tumour growth and metastases. Therefore, we next investigated whether MAO-A has effects on glycolysis in GC. The expression of key glycolytic genes, including hexokinase 2 (HK II) and pyruvate dehydrogenase (PDH) were examined. The results shown in Figure 6A and B indicated that MAO-A knockdown resulted in a decrease in the expression of $\mathrm{HK}$ II and an increase in that of PDH. In addition, we used a Seahorse XFe24 Extracellular Flux Analyzer to monitor extracellular acidification rates, which is an indicator used to estimate glycolysis. The results showed that aerobic glycolysis in AGS cells was blocked by MAO-A RNA interference (Figure 6C) with similar results observed in MGC803 cells (Figure 6D). The metabolic signatures assay and glycolytic enzyme immunoblotting results both indicated that the glycolysis in human gastric tumour cells was mediated by MAO-A.

\section{Discussion}

In the present study, we investigated the role of MAO-A in GC development and in the initiation of mitochondrial metabolic reprogramming. We demonstrated that knockdown of MAO-A in AGS and MGC803 cells synergistically inhibited the cell cycle progression, proliferation and migration. Mechanistically, MAO-A-mediated mitochondrial dysfunction followed by glycolysis activation, promoting the progression of GC.

MAO-A was initially recognized as a potential neuromodulator related to human psychiatric disorders. ${ }^{22}$ Recently, emerging evidence has revealed that MAO-A plays a role in cancers by mediating the epithelial-mesenchymal transition, proliferation and invasion of cancer cells. ${ }^{23,24}$ For example, in human neuroblastoma cells, higher levels of MAO-A results in increased basal ROS levels which promotes autophagy through Bcl-2 phosphorylation. ${ }^{25}$ Liao et al have concluded that sustained inhibition of MAO-A caused by IL-6/ IL-6R activation promotes breast cancer angiogenesis and invasion in hypoxic environment. ${ }^{26}$ Excitingly, a mitochondrial-targeting MAO-A inhibitor was synthesized and used to target prostate cancer, which dramatically inhibited tumour growth. ${ }^{27}$ Our results first showed that expression of MAO-A was significantly increased in vivo in GC tissues compared with that observed in paired adjacent noncancerous tissues. Moreover, the in vitro protein levels of MAO-A were shown to be higher in GC cell lines (AGS, MGC803) than in normal gastric epithelial cells (GES1). Furthermore, MAO-A knockdown in AGS and MGC803 cells caused inhibited cell proliferation and variations in the levels of cyclins associated with the cell cycle, where 
A
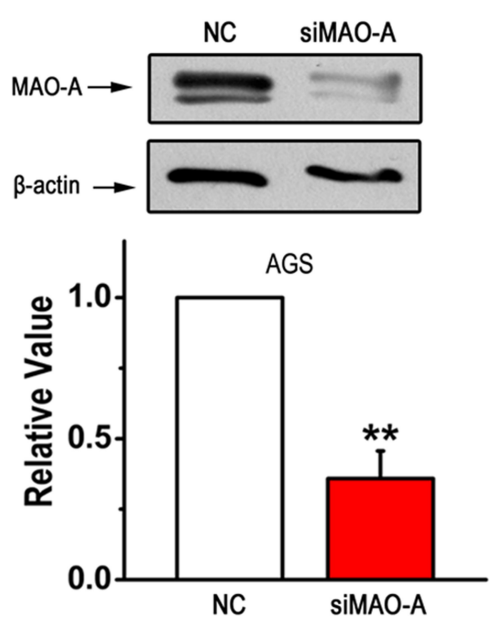

C

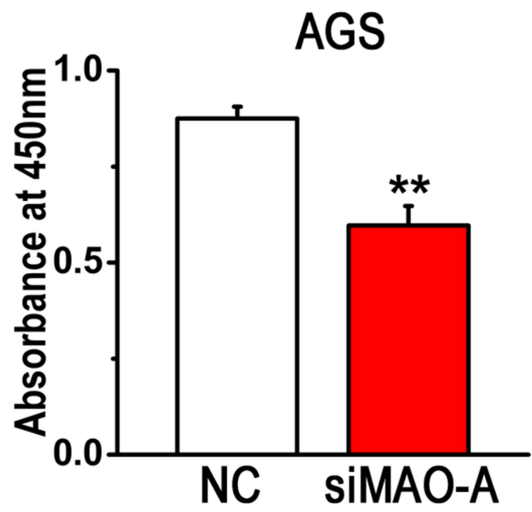

E

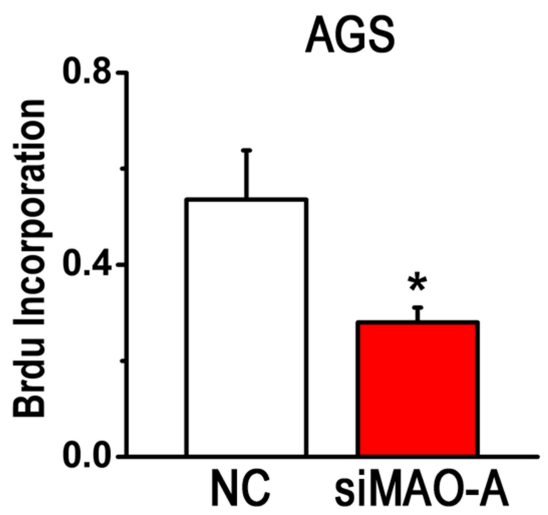

B
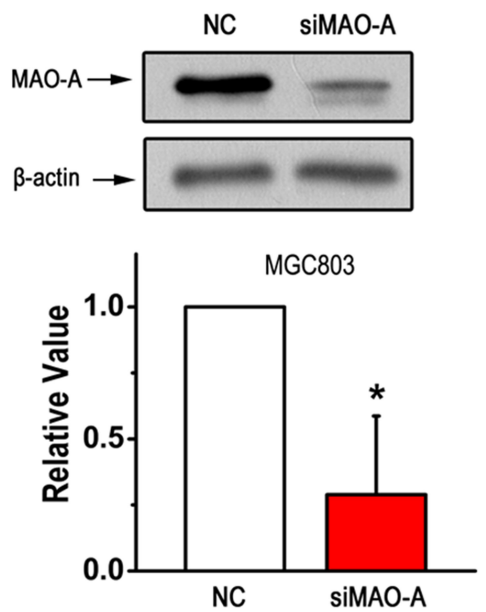

D $\quad$ MGC803

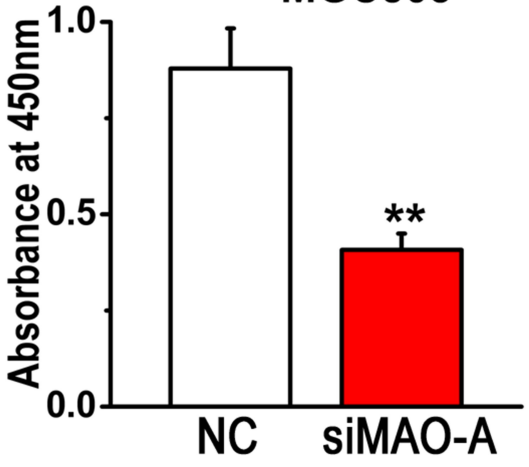

$\mathbf{F}$

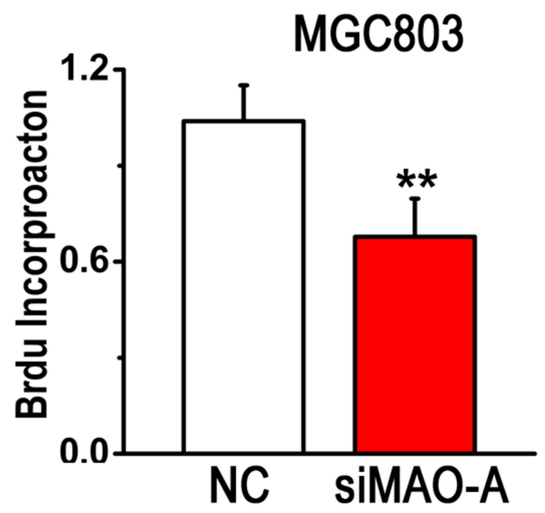

Figure 2 MAO-A deletion in GC cells suppresses cell proliferation. (A-B). Expression of intracellular MAO-A was measured to assess the efficiency and specificity of siRNAs. (C-D). The survival rates of AGS and MGC803 cells was were assessed by CCK8 assays. (E-F). BrdU incorporation assays were performed after blocking MAOA. $n=6$. $* \mathrm{p}<0.05$, **P $<0.01$ compared with NC. All of the values are presented as the means \pm SEM.

Abbreviations: AGS and MGC803, human gastric cancer cell lines; BrdU, 5-bromodeoxyuridine; CCK8, cell counting kit-8; GC, gastric cancer; MAO-A, monoamine oxidase-A; NC, non-targeted control; siMAO-A, small interfering RNA of MAO-A.

decreasing levels of cyclin A and D and of cell cycle arrest were observed. In addition, siMAO-A was also observed to inhibit cell migration and further inhibit the invasion of AGS and MGC803 cells. Taken together, the above findings support the hypothesis that MAO-A is a major contributor to GC.
The effects of mitochondrial dysfunction, including oxidative phosphorylation reduction, reactive oxygen species (ROS) accumulation and vigorous mitochondrial dynamics, has been recently shown to promote the survival, resistance, and metastasis of multiple tumours. ${ }^{28,29}$ Metabolic reprogramming occurs by suppressing mitochondrial oxidative 
A

B
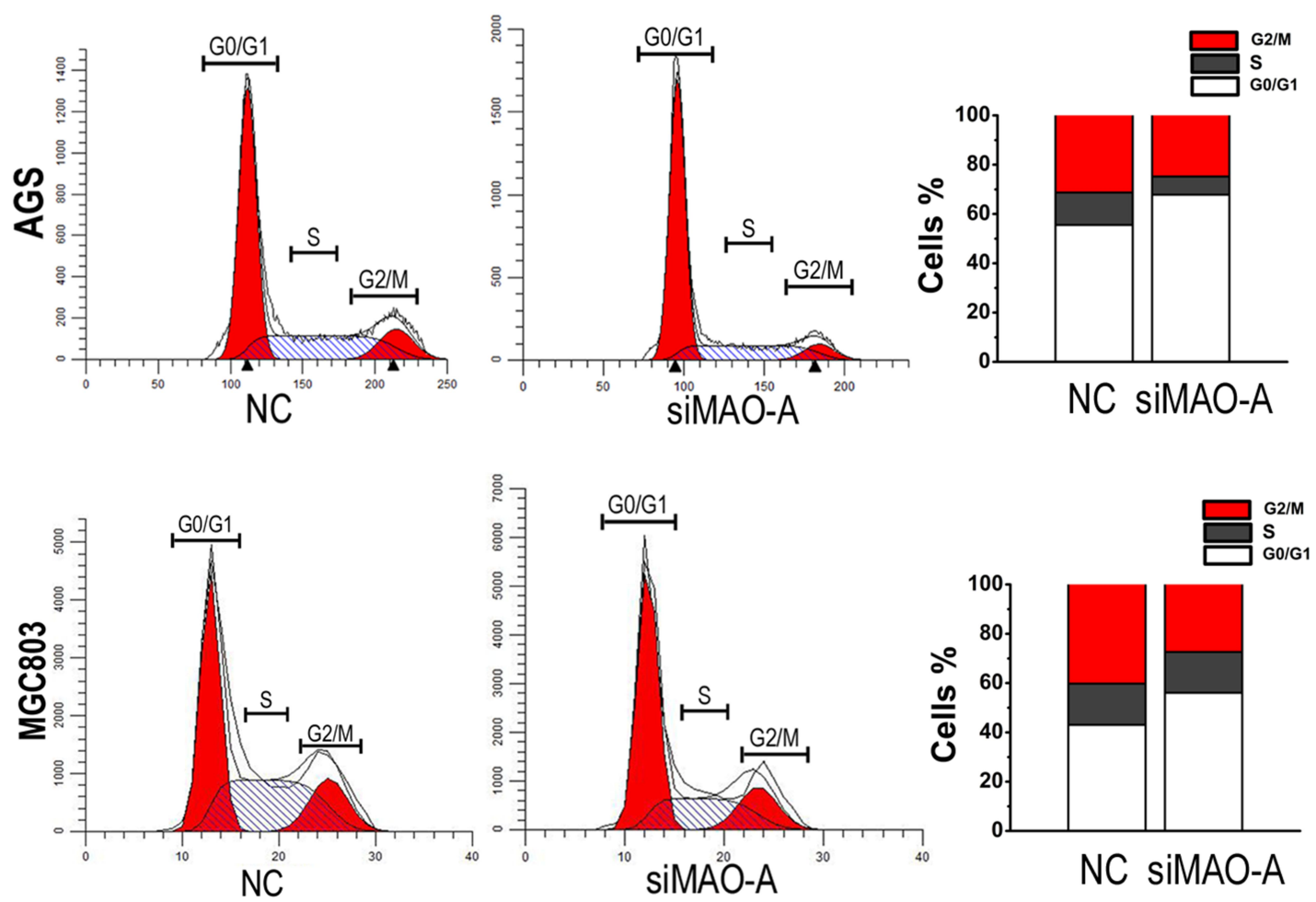

C
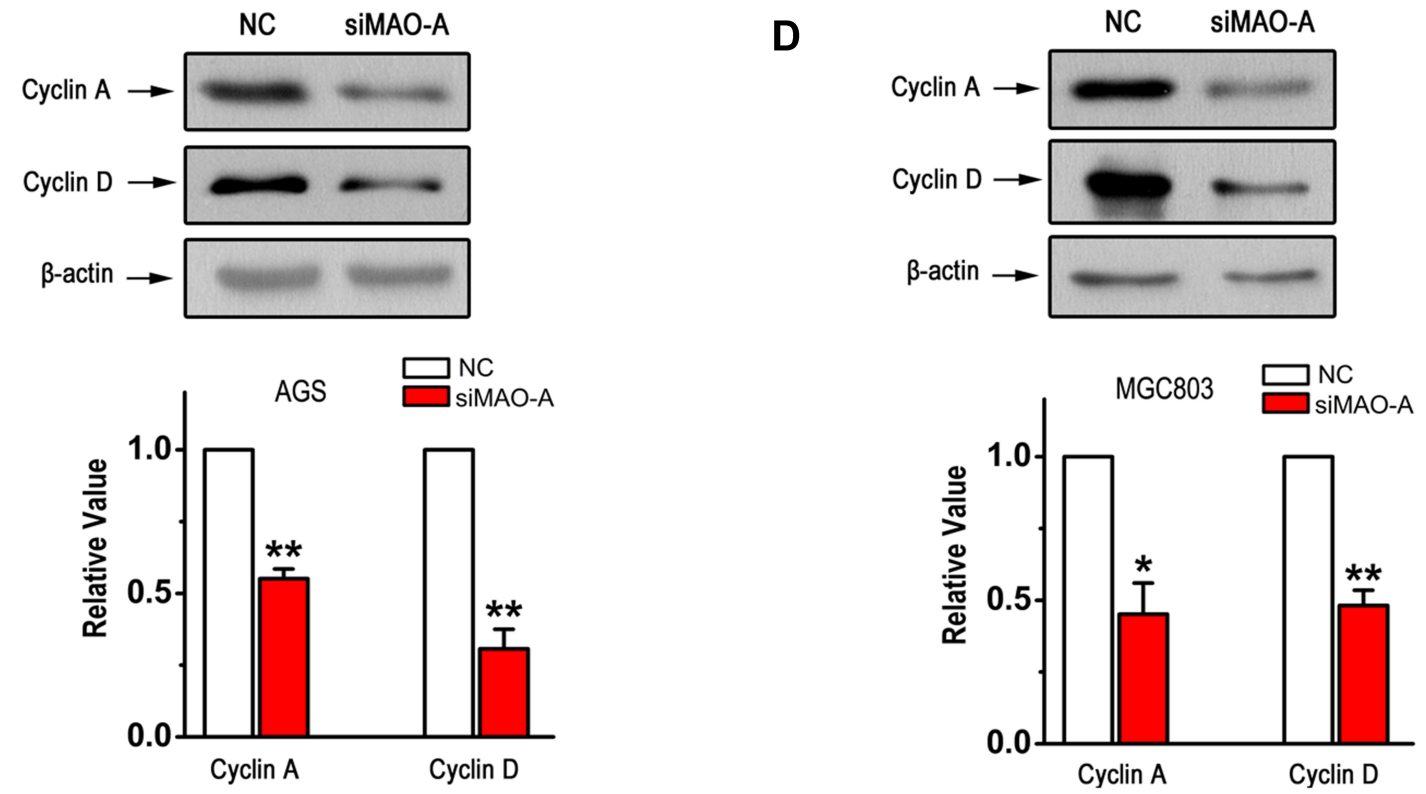

Figure 3 MAO-A plays a role in GC cell cycle progression. (A-B). The number of AGS and MGC803 cells in each phase of the cell cycle was examined by fluorescenceactivated cell sorter analysis. The expression of cyclin A and D in AGS cells (C) and MGC803 cells (D) was measured by Western blot. $n=6$. $* \mathrm{P}<0.05$, **P $<0.0 \mathrm{I}$ compared with NC. All of the values are presented as the means \pm SEM.

Abbreviations: AGS and MGC803, human gastric cancer cell lines; GC, gastric cancer; MAO-A, monoamine oxidase-A; NC, non-targeted control; siMAO-A, small interfering RNA of MAO-A.

phosphorylation (OXPHOS) and switching cellular metabolism towards glycolysis, which results in accumulating lactic acid and facilitates the metastasis of cancer cells. ${ }^{30,31}$
The underlying molecular mechanism of glycolysis in GC may involve the abnormal activity of glycolysis genes, such as increased expression of HK II and the suppression of 
A

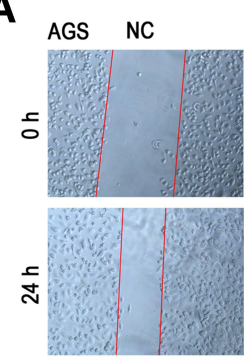

C

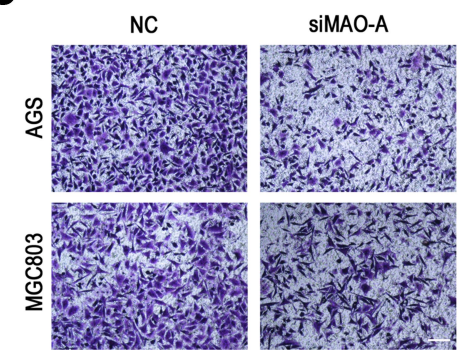

SIMAO-A
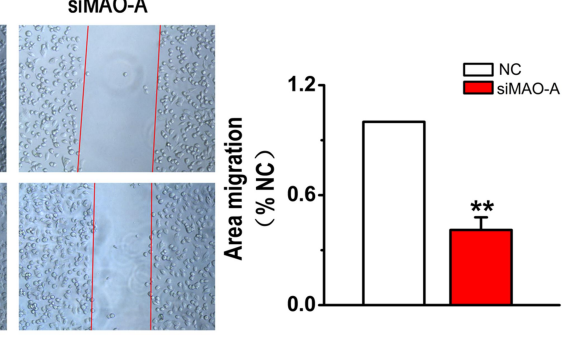

B

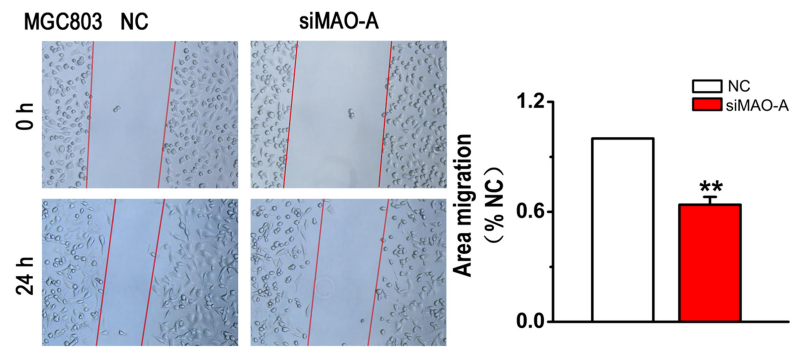

D
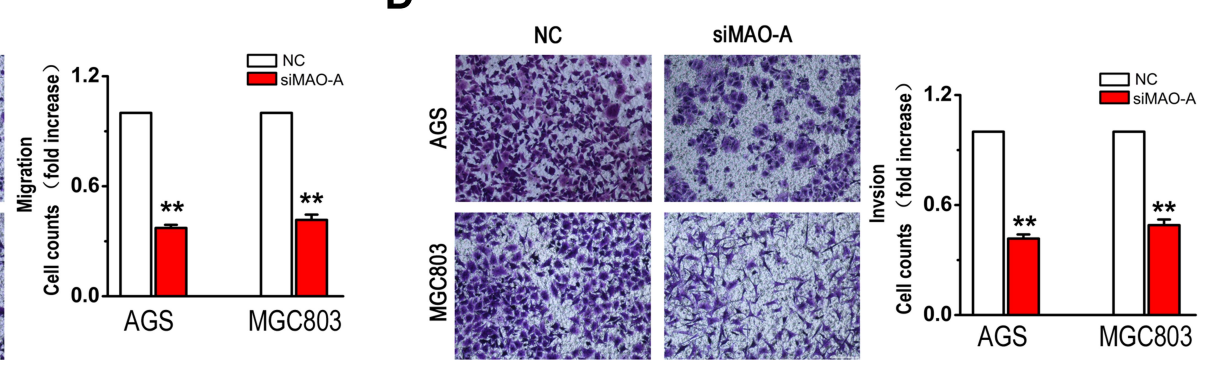

Figure 4 GC cells migration and invasion are mediated by the MAO-A. (A-B). AGS and MGC803 cells were wounded and then treated with NC and siMAO-A for 24 h. (C). AGS and MGC803 cell migration was measured using a Boyden chamber with polycarbonate filters. (D). AGS and MGC803 cell invasion was measured using a Matrigel-coated Boyden chamber with polycarbonate filters. $n=4$. ${ }^{* *} \mathrm{P}<0.01$ compared with NC. All of the values are presented as the means \pm SEM.

Abbreviations: AGS and MGC803, human gastric cancer cell lines; GC, gastric cancer; MAO-A, monoamine oxidase-A; NC, non-targeted control; siMAO-A, small interfering RNA of MAO-A.

A
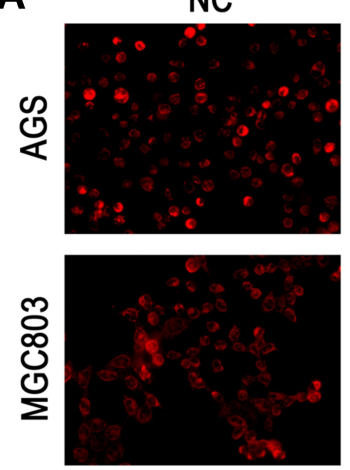

SiMAO-A

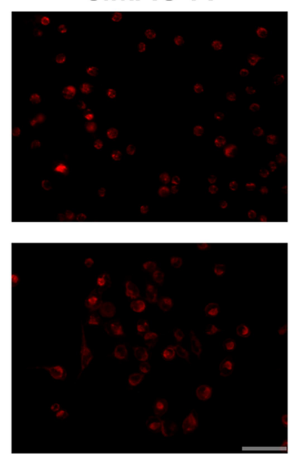

B

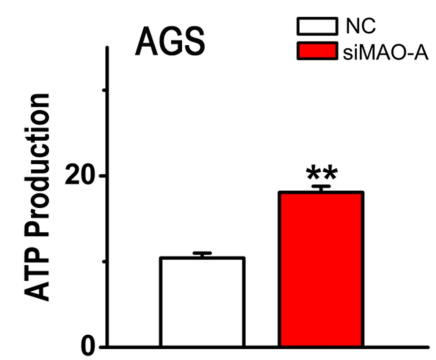

C

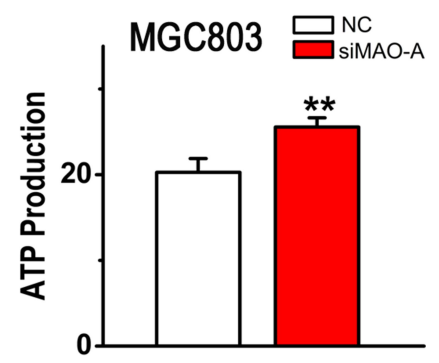

Figure 5 Mitochondrial dysfunction of cultured AGS and MGC803 cells is mediated by MAO-A. (A). MitoSOX Red staining for mitochondrial ROS production in GC cells, Scale bar $=50 \mu \mathrm{m}$. (B-C). MAO-A silencing dramatically increased the ATP level in cultured AGS and MGC803 cells. $n=4$. **P $<0.01$ compared with NC. All of the values are presented as the means \pm SEM.

Abbreviations: AGS and MGC803, human gastric cancer cell lines; GC, gastric cancer; MAO-A, monoamine oxidase-A; NC, non-targeted control; siMAO-A, small interfering RNA of MAO-A.

PDH. ${ }^{32,33}$ Elevated HK II expression in non-small-cell lung cancer, colon cancer, myeloma, breast cancer and pancreatic cancer cells compared with normal tissues has been reported. ${ }^{34,35}$ For example, a high glycolytic rate has been shown to be closely associated with the increased expression of HK II and its binding with voltage-dependent anionic channel (VDAC) in the mitochondria of breast cancer cells, resulting in apoptosis inhibition and chemotherapeutic agent resistance. ${ }^{36}$ In contract, among the different regulators of mitochondrial metabolic function, $\mathrm{PDH}$ is known to modulate pyruvate flux from the cytoplasm to the mitochondria by coupling glycolysis to OXPHOS. ${ }^{37}$ The results of previous studies have suggested that the metabolic changes that occur in multiple human tumour cells may be due to the attenuation of PDH activity. ${ }^{38}$ In the present study, our results demonstrated that MAO-A functions as an essential driver in mitochondrial metabolism, which was proved by measurements of ECAR and the 
A
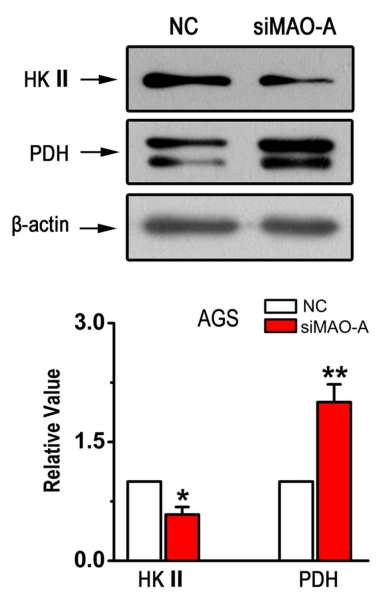

B
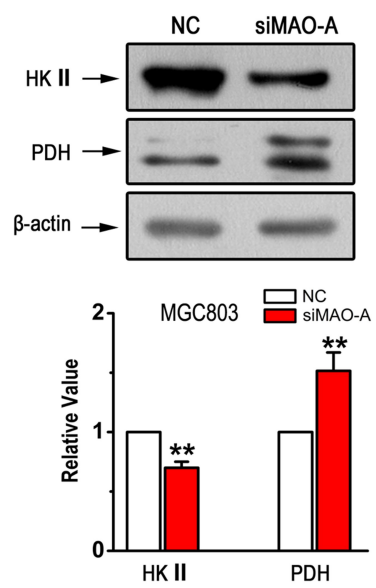

C

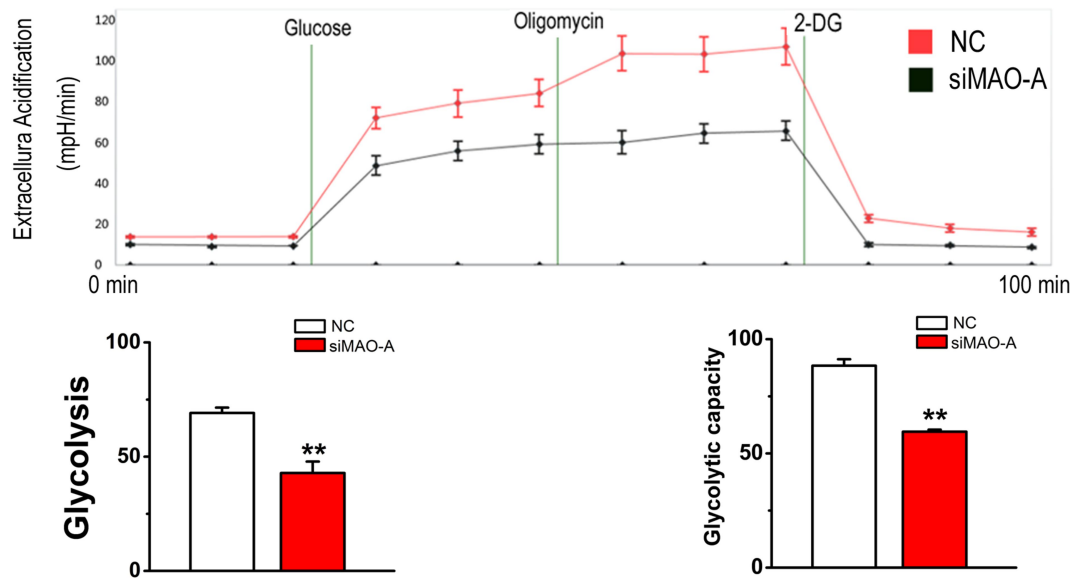

D

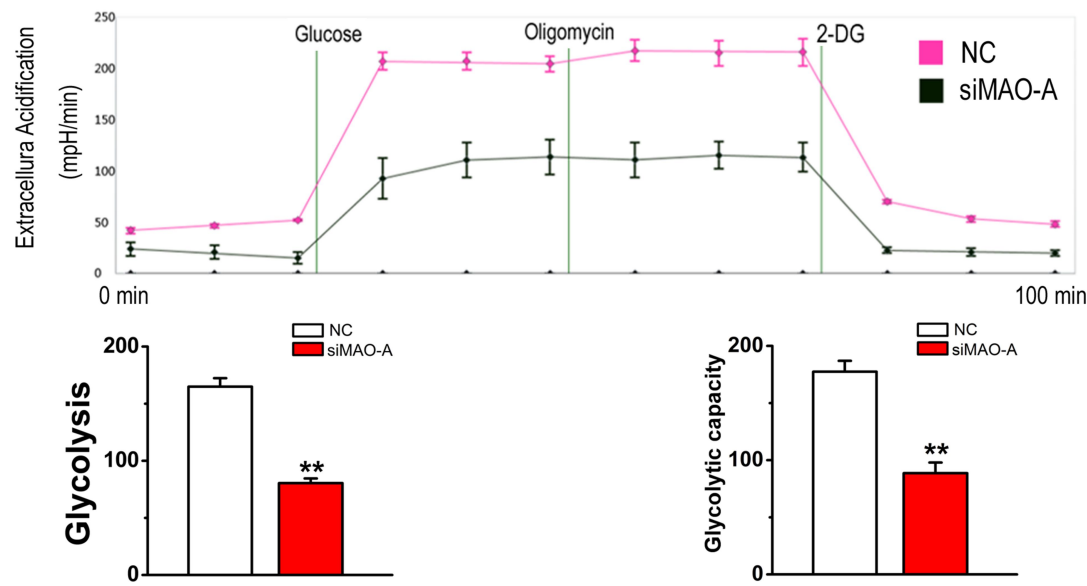

Figure 6 Glycolytic stress in cultured GC cells is relieved by siMAO-A. (A). Western blot analysis of HK II and PDH in AGS cells in the indicated groups. (B). The immunoblotting of HK II and PDH in MGC803 cells after MAO-A knockdown. (C-D). Extracellular acidification rates of cultured AGS and MGC803 cells measured by Seahorse XFe24 to assess glycolysis following $10 \mathrm{mM}$ glucose, I $\mu$ M oligomycin and $100 \mathrm{mM} 2$-DG treatments. $\mathrm{n}=5$. $* \mathrm{P}<0.05$, $* * \mathrm{P}<0.0 \mathrm{I}$ compared with NC. All of the values are presented as the means \pm SEM.

Abbreviations: AGS and MGC803, human gastric cancer cell lines; 2-DG, 2-deoxy-D-glucose; GC, gastric cancer; HK II, hexokinase II; NC, non-targeted control; PDH, pyruvate dehydrogenase; ROS, reactive oxygen species; siMAO-A, small interfering RNA of MAO-A.

expression of glycolytic enzymes (HK II and PDH) as well as observed ATP and ROS production levels. Importantly, since the conversion of pyruvate to acetyl CoA by PDH is a key rate-limiting step that determines the metabolic fate between glycolysis versus OXPHOS,${ }^{37}$ the increased expression of PDH was observed upon silencing of MAOA in both AGS and MGC803 cells, further supporting our hypothesis that MAO-A is a key player in inducing mitochondrial metabolic reprogramming in GC. Thus, our results prove definitive evidence that MAO-A plays a crucial role in both mitochondrial damage and metabolic phenotypic transition, leading to excessive cell proliferation and migration in the pathogenesis of GC.

However, based on the findings of this manuscript, we speculate that MAO-A is not directly involved in the regulation of mitochondrial respiration or glycolysis but rather influences mitochondrial metabolic processes through some unknown signalling pathways or by interacting proteins, such as HK II and PDH. Further investigations are needed to identify the proteins and signalling pathways regulated by MAO-A in GC.

\section{Conclusion}

In summary, for the first time, our results revealed the crucial role of MAO-A in glycolysis and mitochondrial homeostasis during the progression of gastric cancer. The results of the present study not only advance our understanding of the pathogenic effects of MAO-A in the pathogenesis of gastric cancer but also offer a new mechanism to treat this disease in the future. 


\section{Funding}

This work was supported by the Shandong Medical and Health Science and Technology Development Plan Project [2017WSA18029]. The Innovation Project of Shandong Academy of Medical Sciences, Youth Innovation Fund Project of Affiliated Hospital of Shandong Academy of Medical Sciences [2017-03].

\section{Disclosure}

The authors report no conflicts of interest for this work.

\section{References}

1. Kumar S, Metz DC, Ellenberg S, et al. Risk Factors and Incidence of Gastric Cancer After Detection of Helicobacter pylori Infection: A Large Cohort Study. 2020;158(3):527-536 e527.

2. Liu F, Fu J, Bergstrom K, et al. Core 1-derived mucin-type O-glycosylation protects against spontaneous gastritis and gastric cancer. $J$ Exp Med. 2020;217(1):1. doi:10.1084/jem.20182325

3. Zhang Z, Pi J, Zou D, et al. microRNA arm-imbalance in part from complementary targets mediated decay promotes gastric cancer progression. Nat Commun. 2019;10(1):4397. doi:10.1038/s41467019-12292-5

4. Ooi WF, Nargund AM, Lim KJ, et al. Integrated paired-end enhancer profiling and whole-genome sequencing reveals recurrent CCNE1 and IGF2 enhancer hijacking in primary gastric adenocarcinoma. Gut. 2019;2.

5. Xu DH, Li Q, Hu H, et al. Transmembrane protein GRINA modulates aerobic glycolysis and promotes tumor progression in gastric cancer. $J$ Exp Clin Cancer Res. 2018;37(1):308. doi:10.1186/s13046-0180974-1

6. Yu XW, Wei D, Gao YS, et al. Synergistic combination of DT-13 and Topotecan inhibits aerobic glycolysis in human gastric carcinoma BGC-823 cells via NM IIA/EGFR/HK II axis. J Cell Mol Med. 2019;23(10):6622-6634. doi:10.1111/jcmm.14523

7. Cai Z, Zhao JS, Li JJ, et al. A combined proteomics and metabolomics profiling of gastric cardia cancer reveals characteristic dysregulations in glucose metabolism. Mol Cell Proteomics. 2010;9 (12):2617-2628. doi:10.1074/mcp.M110.000661

8. Mao A, Zhou X, Liu Y, Ding J, Miao A, Pan G. KLF8 is associated with poor prognosis and regulates glycolysis by targeting GLUT4 in gastric cancer. J Cell Mol Med. 2019;23(8):5087-5097. doi:10.1111/ jemm. 14378

9. Liu YD, Yu L, Ying L, et al. Toll-like receptor 2 regulates metabolic reprogramming in gastric cancer via superoxide dismutase 2 . Int $J$ Cancer. 2019;144(12):3056-3069. doi:10.1002/ijc.32060

10. Momcilovic M, Jones A, Bailey ST, et al. In vivo imaging of mitochondrial membrane potential in non-small-cell lung cancer. Nature. 2019;575(7782):380-384.

11. Bock FJ, Tait SWG. Mitochondria as multifaceted regulators of cell death. Nat Rev Mol Cell Biol. 2020;21(2):85-100. doi:10.1038/ s41580-019-0173-8

12. Badrinath N, Yoo SY. Mitochondria in cancer: in the aspects of tumorigenesis and targeted therapy. Carcinogenesis. 2018;39 (12):1419-1430. doi:10.1093/carcin/bgy148

13. Guerra F, Arbini AA, Moro L. Mitochondria and cancer chemoresistance. Biochim Biophys Acta Bioenerg. 2017;1858 (8):686-699. doi:10.1016/j.bbabio.2017.01.012

14. Cavalcante GC, Marinho ANR, Anaissi AK, et al. Whole mitochondrial genome sequencing highlights mitochondrial impact in gastric cancer. Sci Rep. 2019;9(1):15716. doi:10.1038/s41598-019-51951-x
15. Liao CP, Lin TP, Li PC, et al. Loss of MAOA in epithelia inhibits adenocarcinoma development, cell proliferation and cancer stem cells in prostate. Oncogene. 2018;37(38):5175-5190. doi:10.1038/s41388018-0325-x

16. Staes N, Sherwood CC, Freeman H, et al. Serotonin Receptor 1A Variation Is Associated with Anxiety and Agonistic Behavior in Chimpanzees. Mol Biol Evol. 2019;36(7):1418-1429. doi:10.1093/ molbev/msz061

17. Nymberg C, Jia $T$, Lubbe $S$, et al. Neural mechanisms of attention-deficit/hyperactivity disorder symptoms are stratified by MAOA genotype. Biol Psychiatry. 2013;74(8):607-614. doi:10.10 16/j.biopsych.2013.03.027

18. Haberstick BC, Lessem JM, Hewitt JK, et al. MAOA genotype, childhood maltreatment, and their interaction in the etiology of adult antisocial behaviors. Biol Psychiatry. 2014;75(1):25-30. doi:10.1016/j.biopsych.2013.03.028

19. Wu JB, Lin TP, Gallagher JD, et al. Monoamine oxidase A inhibitor-near-infrared dye conjugate reduces prostate tumor growth. $J$ Am Chem Soc. 2015;137(6):2366-2374. doi:10.1021/ja512613j

20. Li J, Yang XM, Wang YH, et al. Monoamine oxidase A suppresses hepatocellular carcinoma metastasis by inhibiting the adrenergic system and its transactivation of EGFR signaling. $J$ Hepatol. 2014;60 (6):1225-1234. doi:10.1016/j.jhep.2014.02.025

21. Koppenol WH, Bounds PL, Dang CV. Otto Warburg's contributions to current concepts of cancer metabolism. Nat Rev Cancer. 2011;11 (5):325-337. doi:10.1038/nrc3038

22. Wagels L, Votinov M, Kellermann T, et al. Exogenous testosterone and the monoamine-oxidase A polymorphism influence anger, aggression and neural responses to provocation in males. Neuropharmacology. 2019;156:107491. doi:10.1016/j.neuropharm.2019.01.006

23. Yang XG, Mou YH, Wang YJ, et al. Design, Synthesis, and Evaluation of Monoamine Oxidase A Inhibitors(-)Indocyanine Dyes Conjugates as Targeted Antitumor Agents. Molecules. 2019;24:7.

24. Liu F, Hu L, Ma Y, et al. Increased expression of monoamine oxidase A is associated with epithelial to mesenchymal transition and clinicopathological features in non-small cell lung cancer. Oncol Lett. 2018;15(3):3245-3251.

25. Ugun-Klusek A, Theodosi TS, Fitzgerald JC, et al. Monoamine oxidase-A promotes protective autophagy in human SH-SY5Y neuroblastoma cells through Bcl-2 phosphorylation. Redox Biol. 2019;20:167-181. doi:10.1016/j.redox.2018.10.003

26. Bharti R, Dey G, Das AK, Mandal M. Differential expression of IL-6/IL-6R and MAO-A regulates invasion/angiogenesis in breast cancer. Br J Cancer. 2018;118(11):1442-1452. doi:10.1038/s41416018-0078-x

27. Lv Q, Yang X, Wang M, et al. Mitochondria-targeted prostate cancer therapy using a near-infrared fluorescence dye-monoamine oxidase A inhibitor conjugate. J Control Release. 2018;279:234-242. doi:10. 1016/j.jconrel.2018.04.038

28. Moro L. Mitochondrial Dysfunction in Aging and Cancer. J Clin Med. 2019;8(11):11. doi:10.3390/jcm8111983

29. Dai W, Jiang L. Dysregulated Mitochondrial Dynamics and Metabolism in Obesity, Diabetes, and Cancer. Front Endocrinol (Lausanne). 2019;10:570. doi:10.3389/fendo.2019.00570

30. Tang Z, Li L, Tang Y, et al. CDK2 positively regulates aerobic glycolysis by suppressing SIRT5 in gastric cancer. Cancer Sci. 2018;109(8):2590-2598. doi:10.1111/cas.13691

31. Michelakis ED, Sutendra G, Dromparis P, et al. Metabolic modulation of glioblastoma with dichloroacetate. Sci Transl Med. 2010;2 (31):31ra34. doi:10.1126/scitranslmed.3000677

32. Liu Z, Yu M, Fei B, Fang X, Ma T, Wang D. miR215p targets PDHA1 to regulate glycolysis and cancer progression in gastric cancer. Oncol Rep. 2018;40(5):2955-2963.

33. Sun K, Hu P, Xu F. LINC00152/miR-139-5p regulates gastric cancer cell aerobic glycolysis by targeting PRKAA1. Biomed Pharmacother. 2018;97:1296-1302. doi:10.1016/j.biopha.2017.11.015 
34. Zhao W, Li W, Dai W, Huang N, Qiu J. LINK-A promotes cell proliferation through the regulation of aerobic glycolysis in non-small-cell lung cancer. Onco Targets Ther. 2018;11:6071-6080. doi:10.2147/OTT.S171216

35. Song W, Wang Z, Gu X, et al. TRIM11 promotes proliferation and glycolysis of breast cancer cells via targeting AKT/GLUT1 pathway. Onco Targets Ther. 2019;12:4975-4984. doi:10.2147/OTT.S207723

36. Guo Y, Wei L, Zhou Y, et al. Flavonoid GL-V9 induces apoptosis and inhibits glycolysis of breast cancer via disrupting GSK-3betamodulated mitochondrial binding of HKII. Free Radic Biol Med. 2020;146:119-129. doi:10.1016/j.freeradbiomed.2019.10.413
37. Lee M, Yoon JH. Metabolic interplay between glycolysis and mitochondrial oxidation: the reverse Warburg effect and its therapeutic implication. World J Biol Chem. 2015;6(3):148-161. doi:10.4331/ wjbc.v6.i3.148

38. Yonashiro R, Eguchi K, Wake M, Takeda N, Nakayama K. Pyruvate dehydrogenase PDH-E1beta controls tumor progression by altering the metabolic status of cancer cells. Cancer Res. 2018;78 (7):1592-1603. doi:10.1158/0008-5472.CAN-17-1751

\section{Publish your work in this journal}

Cancer Management and Research is an international, peer-reviewed open access journal focusing on cancer research and the optimal use of preventative and integrated treatment interventions to achieve improved outcomes, enhanced survival and quality of life for the cancer patient.
The manuscript management system is completely online and includes a very quick and fair peer-review system, which is all easy to use. Visit http://www.dovepress.com/testimonials.php to read real quotes from published authors. 